\title{
The spectroscopic orbits and physical parameters of GG Car
}

\author{
Paula E. Marchiano ${ }^{1}$, Elisande Brandi $i^{1,2,3}$, Maria F. Muratore ${ }^{1}$, \\ Claudio Quiroga ${ }^{1,2}$, Osvaldo Ferrer ${ }^{1,4}$ and Lia García ${ }^{1,2}$ \\ ${ }^{1}$ Facultad de Ciencias Astronómicas y Geofísicas, Universidad Nacional de La Plata, \\ Paseo del Bosque, S/N, 1900 La Plata, Argentina \\ email: pmarchiano@fcaglp.unlp.edu.ar \\ ${ }^{2}$ Instituto de Astrofísica La Plata, CCT La Plata, CONICET \\ ${ }^{3}$ Comisión de Investigaciones Científicas de la Prov. de Buenos Aires (CIC) \\ ${ }^{4}$ Consejo Nacional de Investigaciones Científicas y Técnicas (CONICET)
}

\begin{abstract}
GG Car is a peculiar B type star with emission lines classified as a B[e] supergiant star. In this work we present a spectral analysis of this system based on spectra obtained at Casleo. We fit the spectral energy distribution adopting a model for the gas and dust circumstellar components and thus we obtain the physical parameters of the star and its environment.
\end{abstract}

Keywords. stars: individual (GG Car), stars: emission-line, stars: fundamental parameters

\section{Introduction}

Hernandez et al. (1981) were the first to investigate the possible periodic variability of the spectrum of GG Car. Gosset et al. (1985) used photoelectric photometry in both the standard UBV and Stromgren uvby systems and they determined two possibles periods: 31,020 and 62,039 days, then the first value was definitely confimed from the radial velocities with spectroscopic data along the light curve. Finally, it is known that GG Car has an infrared excess of the type that appears to be associated with circumstellar dust. From IUE low resolution data, Brandi et al. (1987) propose a value of the B-V excess of $E_{B-V}=0,52 \pm 0,04$.

\section{The spectroscopy orbits and the spectral distribution}

The observational material consists of 55 spectra which were obtained with the $2.15 \mathrm{~m}$ "Jorge Sahade" telescope at CASLEO (San Juan, Argentina). An echelle spectrograph REOSC and a teck CCD detector were used, resulting a resolution $R \sim 12000$ and an spectral range of 4000 to $7100 \AA$ and 5000 to $8700 \AA$.

The He I $(\lambda \lambda 4471,5875,6678,7065)$ line profiles show a large variability and several absorption components were detected. One of these components corresponds to the blueshifted absorption of a P-Cyni profile. Our task was to calculate the radial velocities of the other components and the result of this analysis led us to find that they could be associated with the two stars of the binary system.

Table 1 shows the orbital elements obtained for combined solutions. Fig. 1 Left shows the radial velocity curves obtained for both components of the system. Continuous curves give the result of the best fit with $P=31$ days.

In order to reproduce the continuum energy distribution of GG Car (Fig. 1 Right), we adopt B-type flux models (Kurucz, 1979) surrounded by a gaseous circumstellar envelope and outermost dust layers. Taking into account the classification criterion of Lopes et al. 
Table 1. Orbital solutions obtained from the HeI absorption lines 4471, 5875, 6678 and $7065 \AA$

\begin{tabular}{|c|c|c|c|c|c|c|c|c|c|c|}
\hline Comp. & $\begin{array}{c}\mathrm{P} \\
{[\text { days }]}\end{array}$ & $\begin{array}{c}K \\
{\left[\mathrm{kms}^{-1}\right]}\end{array}$ & $\begin{array}{c}\gamma_{0} \\
{\left[\mathrm{kms}^{-1}\right]}\end{array}$ & e & $\begin{array}{c}\omega \\
{[\mathrm{deg}]}\end{array}$ & $\begin{array}{c}T_{\circ}^{(1)} \\
{[\mathrm{JD} 24 \ldots]}\end{array}$ & $\begin{array}{c}\mathrm{T}_{c o n j}^{(2)} \\
{[\mathrm{JD} 24 \ldots]}\end{array}$ & $\begin{array}{l}\Delta T^{(3)} \\
{[\text { days }]}\end{array}$ & $\begin{array}{c}a \sin i \\
{[\mathrm{AU}]}\end{array}$ & $\begin{array}{c}M \sin ^{3} \mathrm{i} \\
{\left[\mathrm{M}_{\odot}\right]}\end{array}$ \\
\hline Prim. & $\begin{array}{c}31.033 \pm \\
0.008\end{array}$ & $65.8 \pm 7.3$ & $\begin{array}{c}-162.1 \pm \\
4.3\end{array}$ & $\begin{array}{c}0.28 \pm \\
0.06\end{array}$ & $\begin{array}{c}272 \pm \\
12\end{array}$ & 52020.96 & 52051.93 & 2.44 & $0.18 \pm 0.02$ & $18 \pm 3$ \\
\hline Sec. & & $143.3 \pm 8.7$ & & & & & & & $0.39 \pm 0.02$ & $8 \pm 2$ \\
\hline
\end{tabular}

${ }^{(1)} \mathrm{T}_{\circ}$ : time of periastron passage.

${ }^{(2)} \mathrm{T}_{\text {conj }}$ : time of spectroscopic conjunction.

(3) $\Delta T=\mathrm{T}_{\text {conj }}-\mathrm{T}_{\text {phot-min }}$.

$\mathrm{T}_{\text {phot-min }}=2444260.21$ is the mean epoch of the minimum (Min I) given by Gosset et al. (1985).

The orbital phases were calculated with the ephemeris: $T_{c o n j}=2452051.93+31.033 E$.
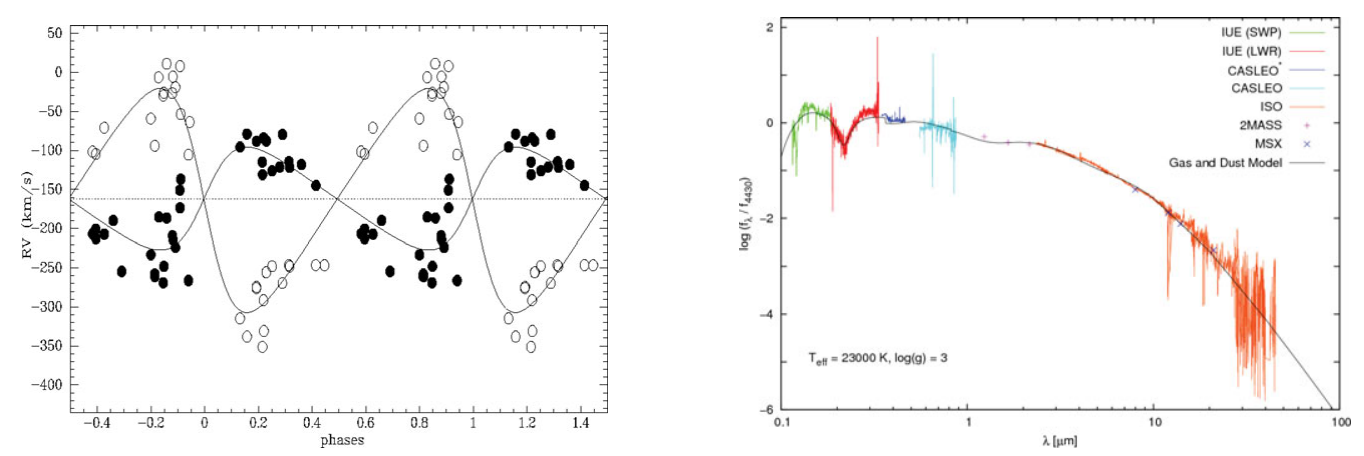

Figure 1. Left: Fill circle: primary component. Empty circle: secondary component. Continuous curve: the best fit of the orbital solutions. Right: The observed energy distribution using available photometric and spectroscopic data and the theoretical model (see Muratore et al., these proceedings.)

(1992) and Clark et al. (2000) together with the calibration temperature scale derived by Zorec et al. (2009), we conclude that the star is consistent with a B0-B2 spectral type.

\section{Conclusions}

We have determined the orbit for both components of the binary system GG Car through a detailed study of the He I lines and we have confirmed the photometric period. The orbital parameters are indicated in Table 1 and a mass ratio $q \cong 2,179$ was obtained.

We have modeled GG Car as a central star with $T_{\text {eff }}=23,000 \mathrm{~K}$ and $\log (g)=3$ surrounded by a spherical envelope consisting of two regions: a layer close to the central star of 3,5 stellar radius composed of ionized gas and other outermost layers composed of dust with $E_{B-V}=0,3$. For the interstellar medium, an $E_{B-V}=0,18$ was added.

\section{References}

Brandi, E., Gosset, E., \& Swings, J.-P. 1987, A\&A 175, 151

Clark, J. S. \& Steele, I. A. 2000, $A \& A S$ 141, 65

Gosset, E., Hutsemekers, D., Swings, J. P., \& Surdej, J. 1985, A\&A 153, 71

Hernández, C. A., Lopez, L., Sahade, J., Thackeray, A. D. 1981, PASP 93, 747

Kurucz, R. L. 1979, ApJS 40, 1

Lopes, D. F., Damineli Neto, A., \& de Freitas Pacheco, J. A. 1992, A\& A 261, 482

Zorec, J., Cidale, L., Arias, M. L., \& Frémat, Y. et al. 2009, A\&̋A 501, 297 